\title{
CHRNA7 triplication associated with cognitive impairment and neuropsychiatric phenotypes in a three-generation pedigree
}

\author{
Claudia Soler-Alfonso', Claudia MB Carvalho ${ }^{2,3}$, Jun $\mathrm{Ge}^{2}$, Erin K Roney ${ }^{2}$, Patricia I Bader ${ }^{4}$, \\ Katarzyna E Kolodziejska², Rachel M Miller ${ }^{4}$, James R Lupski ${ }^{2,5}$, Pawel Stankiewicz ${ }^{2}$, \\ Sau Wai Cheung ${ }^{2}$, Weimin $\mathrm{Bi}^{2}$ and Christian P Schaaf ${ }^{\star, 2,6}$
}

\begin{abstract}
Although deletions of CHRNA7 have been associated with intellectual disability (ID), seizures and neuropsychiatric phenotypes, the pathogenicity of CHRNA7 duplications has been uncertain. We present the first report of CHRNA7 triplication. Three generations of a family affected with various neuropsychiatric phenotypes, including anxiety, bipolar disorder, developmental delay and ID, were studied with array comparative genomic hybridization (aCGH). High-resolution aCGH revealed a 650-kb triplication at chromosome 15q13.3 encompassing the CHRNA7 gene, which encodes the alpha7 subunit of the neuronal nicotinic acetylcholine receptor. A small duplication precedes the triplication at the proximal breakpoint junction, and analysis of the breakpoint indicates that the triplicated segment is in an inverted orientation with respect to the duplication. CHRNA7 triplication appears to occur by a replication-based mechanism that produces inverted triplications embedded within duplications. Co-segregation of the CHRNA7 triplication with neuropsychiatric and cognitive phenotypes provides further evidence for dosage sensitivity of CHRNA7.
\end{abstract}

European Journal of Human Genetics (2014) 22, 1071-1076; doi:10.1038/ejhg.2013.302; published online 15 January 2014

Keywords: dosage sensitivity; copy number variation; cholinergic nervous system; autism spectrum disorder

\section{INTRODUCTION}

Copy number variants (CNVs) of chromosome 15q13.3 have been associated with multiple neurological and neuropsychiatric phenotypes. ${ }^{1}$ In general, there are two different sizes of recurrent nonallelic homologous recombination (NAHR)-mediated deletions and duplications at this locus: the larger CNVs utilize BP4 and BP5 as substrate pairs, and typically involve a $1.6-\mathrm{Mb}$ genomic interval, whereas the smaller CNVs arise between two CHRNA7-low copy repeat (LCR) copies, and are 350-680 kb in size. Although the larger deletion and duplication harbor six RefSeq genes (MTMR15, MTMR10, TRPM1, KLF13, OTUD7A, and CHRNA7) and one miRNA (hsa-mir211), the smaller deletion and duplication CNV encompass only the entire CHRNA7 gene and the first exon of a non-coding RNA-variant of OTUD7A. The BP4 and BP5 microdeletion has been found in patients with developmental delay/intellectual disability (DD/ID), epilepsy, autism, schizophrenia and other neurocognitive disorders. ${ }^{1,2}$ CHRNA7 was considered the likely culprit gene to cause neurological phenotypes in patients with the 15q13.3 deletion, which was subsequently confirmed by the identification of the small $(680 \mathrm{~kb})$ CHRNA7 deletion, manifesting a similar phenotypic spectrum as the larger deletion. ${ }^{3}$ Follow-up studies have strongly associated $15 q 13.3$ deletions with both $\mathrm{DD} / \mathrm{ID}^{4}$ and schizophrenia. ${ }^{5}$

The reciprocal microduplications of $15 \mathrm{q} 13.3$, which are very common in the general population with an estimated frequency of
1 in $180,{ }^{6}$ have been more challenging to interpret, as they do not manifest the high penetrance of neuropsychiatric phenotypes seen in deletion cases. Van Bon et al first reported four patients with cognitive impairment and psychiatric disorders coinciding with BP4 and BP5 duplications. This was followed by the description of 55 cases of smaller-sized CHRNA7 duplications (350-680 kb in size). ${ }^{7}$ The latter report provided clinical data and pedigree analysis of 11 families and suggested that the duplication may be associated with DD, ID, and a variety of neuropsychiatric phenotypes, but with high variability in expressivity and reduced penetrance. Additional studies showed that 15q13.3 duplications were not associated with schizophrenia, ${ }^{5}$ but significantly associated with DD and ID. ${ }^{4}$ Furthermore, the small CHRNA7 duplication was found as a risk factor for attention deficit hyperactivity disorder. ${ }^{8}$

Here, we report triplication of CHRNA7, segregating in four affected family members in three generations (Figure 1). We present a detailed clinical and behavioral characterization of the affected individuals.

\section{Clinical description}

The propositus (III-2) was a 10-year-old boy at the time he presented to the genetics clinic for evaluation of ID and autistic behaviors. The boy was born as a monozygotic twin conceived naturally by a 26-year-old mother and 30-year-old father. Both parents were

${ }^{1}$ Department of Pediatrics, Division of Medical Genetics, University of Texas Health Science Center, Houston, TX, USA; ${ }^{2}$ Department of Molecular and Human Genetics, Baylor College of Medicine, Houston, TX, USA; ${ }^{3}$ Centro de Pesquisas René Rachou - FIOCRUZ, Belo Horizonte, Brazil; ${ }^{4}$ Parkview Health Laboratories, Genetics Center, Fort Wayne, IN, USA; ${ }^{5}$ Department of Pediatrics, Texas Children's Hospital, Houston, TX, USA; ${ }^{6}$ The Jan and Dan Duncan Neurological Research Institute, Texas Children's Hospital, Houston, TX, USA

*Correspondence: Dr CP Schaaf, The Jan and Dan Duncan Neurological Research Institute, Texas Children's Hospital, 1250 Moursund Street, Suite 1350 , Houston, TX 77030 , USA. Tel: +1 832824 8787; Fax: +1 832825 1251; E-mail: Schaaf@bcm.edu

Received 26 July 2013; revised 30 October 2013; accepted 27 November 2013; published online 15 January 2014 

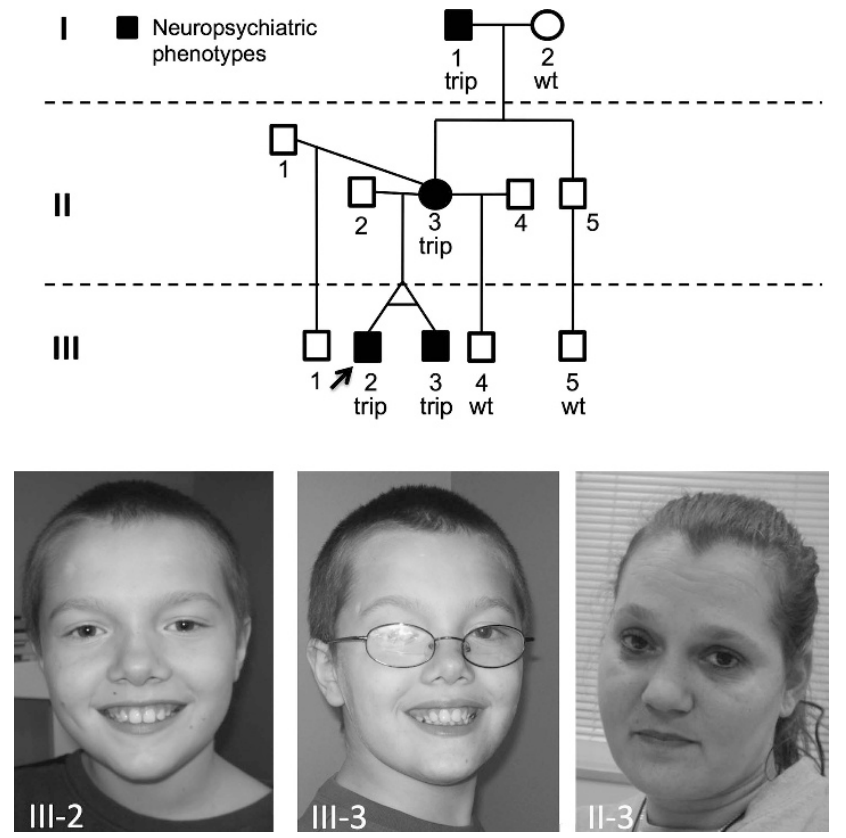

Figure 1 Three generation family with CHRNA7 triplication. Trip, CHRNA7 triplication; wt, wildtype (tested for CHRNA7 copy number by FISH, with normal result). Patient photographs: individuals $\mathrm{III}-2$ and III-3 at 10 years of age, individual II-3 at 37 years of age.

of Caucasian descent. The twins were born by spontaneous vaginal delivery at approximately 36 weeks of gestation. The proband's birth weight was $1.955 \mathrm{~kg}$ (10th-25th percentile). The birth head circumference and length were not available for review. He was hospitalized in the neonatal intensive care unit for 1 week because of mild feeding difficulties. Infancy and early childhood were significant for speech delay, with active intervention speech therapy started at 2 years of age, and cognitive impairment. Motor development was reported as normal. At the time of examination, he was in the fourth grade, enrolled in general and special education classes. He was able to follow two-step commands, but required constant repetition and redirection. A short attention span had been noted, as well as low frustration tolerance. He had transition difficulties and did not tolerate changes in his routine. His emotional profile was significant for anxiety and depression. A formal developmental and neuropsychological assessment was performed at 10 years and 7 months of age and was consistent with dysthymia, anxiety disorder, pervasive developmental disorder not otherwise specified (PDD-NOS) and cognitive abilities in the borderline range (full scale IQ of 75 on the Wechsler Intelligence Scale for Children IV).

Upon physical examination, his head circumference was $55 \mathrm{~cm}$ (80th percentile), height was $145 \mathrm{~cm}$ (90th percentile) and weight was $41 \mathrm{~kg}$ (90th percentile). The patient was mildly dysmorphic with a triangular face, hypertelorism (inner canthal distance $4 \mathrm{~cm},>97 \mathrm{th}$ percentile), bilateral prominent antihelices, a broad philtrum and a high-arched palate (Figure 1, III-2). Examination of the extremities revealed bilateral clinodactyly of the fifth fingers, a single palmar crease on the left and generalized joint hypermobility. Neurological examination was abnormal for mild degree of dysarthria and generalized hypotonia. He exhibited poor balance and poor coordination when walking, but was not frankly ataxic. MRI of the brain performed at 10 years of age showed no anatomic abnormalities.

The proband's monozygotic twin brother (III-3) also presented to clinic for an evaluation of DD and learning problems. At the time of evaluation, he was 10 years old and was able to follow one-, but not two-step commands. He was enrolled in special education classes. A short attention span, impulsive behavior and aggressiveness to his classmates had been reported. Like his brother, he did not tolerate changes in his routine. A formal neuropsychological evaluation revealed dysthymia, anxiety disorder, PDD-NOS, language processing deficits, and borderline intellectual functioning (full scale IQ of 84 on the Wechsler Intelligence Scale for Children IV), with significantly more salient non-verbal than verbal functions, impaired language functions, impaired memory functions and impaired cognitive flexibility. On physical examination, he manifested mild dysmorphic features similar to his twin brother (Figure 1, III-3), had mild dysarthria, some coordination difficulties, and generalized hypotonia. At 2 years of age, a head CT had been obtained after accidental head trauma, revealing a left middle cranial fossa arachnoid cyst, which was later confirmed by MRI at 10 years. Routine genetic evaluation and diagnostic testing for both twin brothers included chromosome analysis, FISH analysis for Sotos syndrome, PTEN sequencing, and Fragile $\mathrm{X}$ testing.

The twins' mother (II-3) was 37 years old at the time of examination. She had a history of DD and did not walk until 2-3 years of age. Her medical history was significant for bipolar disorder, anxiety disorder, attention deficit disorder, learning disability, and seizures since her twenties. At the time of examination, she lived in an assisted living facility. Her physical examination was remarkable for bilateral prominent antihelices, bulbous nasal tip, mildly flat philtrum and a fine resting tremor (Figure 1, II-3). The twins' half siblings (III-1 and III-4) were reported as healthy with normal physical appearance, and normal development. Both attended regular schools with no learning difficulties. Individuals II-2 (the twins' father) and I-2 (maternal grandmother) were in good physical health, with no cognitive impairment and no history of neuropsychiatric disease. Clinical information on the twins' maternal grandfather (I-1) is very limited, but significant for alcoholism, drug addiction, and abusive behaviors to family members. Reportedly, he had multiple family members with a history of alcoholism.

\section{METHODS}

The index case was referred to the Medical Genetics Laboratories at Baylor College of Medicine (BCM), Houston, TX, USA, for clinical array comparative genomic hybridization (aCGH) analysis. DNA was extracted from whole blood using the Puregene DNA extraction kit (Gentra, Minneapolis, MN, USA) according to the manufacturer's instructions. The Institutional Review Board of BCM approved the study, and family members were recruited after informed consent was obtained. Photographs of the patients were collected after obtaining informed consent for publication of the photographs in the medical literature.

\section{aCGH analysis and fluorescence in situ hybridization}

Custom aCGH (CMA V8.1 OLIGO) was performed as previously described. ${ }^{9} 10$ Dual-color FISH analysis was performed on cultured lymphoblast cells with the test probe directly labeled by rhodamine (red) and the control probe directly labeled by FITC (green). For each sample, 50 interphase nuclei were examined to evaluate copy number. For FISH analyses using fosmid probes on proband, twin brother and an unaffected control, $>10$ metaphase cells were examined in addition to 50 interphase cells.

Whole-genome high-resolution oligonucleotide aCGH analysis was performed on the proband's (III-2) DNA for confirmation of the triplication and fine structure mapping of the sizes of the triplication, using the NimbleGen array with $4.2 \mathrm{~m}$ oligonucleotides (NimbleGen Systems, Madison, WI, USA), in accordance with the manufacturer's instructions. ${ }^{10}$ 


\section{Breakpoint identification by PCR and sequencing}

To further determine the size, genomic extent and gene content of this rearrangement, we designed a tiling-path oligonucleotide microarray spanning $2.8 \mathrm{Mb}$ of the 15q13.3 region (all coordinates based on hg19): chr15:g.30 200 000-33 000000 that includes BP4 (chr15:g.30370 111-30 935 159) and BP5 (chr15:g.32 $444018-32914165$ ). The custom $4 \times 44 \mathrm{k}$ Agilent Technologies microarray was designed using the Agilent eArray website (http://earray.chem. agilent.com/earray/). We selected 14765 probes, which represents an average distribution of 1 probe per $190 \mathrm{bp}$. Probe labeling and hybridization were performed as described. ${ }^{11}$

Reverse and forward primer pairs (relative to the reference genome) were designed at the apparent boundaries of each duplicated or triplicated segment as defined by aCGH analysis. Long-range PCR was performed using TaKaRa LA Taq (Clontech, Mountain View, CA, USA). PCR sample-specific products for one of the junctions were obtained using the following primers: R2.1 $5^{\prime}$-TGCGATGCAATTGATAGAGC-3' and R1.1 5'-TCACCCATTGCTAGT GCAAC- $3^{\prime}$. Products were sequenced by the Sanger sequencing methodology. PCR and sequencing results were independently confirmed by repeated experiments.

\section{Real-time quantitative PCR (qPCR)}

To assess the copy numbers of regions of interest within 15q13.3, we performed qPCR using PrimeTime Assays (Integrated DNA Technologies, Coralville, IA, USA). The primers and probes for the qPCR reactions were as follows: CHRNA7 exon2 (chr15:g.32323092-32323210) forward 5'-CTCC TTAAGTGTCCCTGCAAG-3' ${ }^{\prime}$, reverse 5'-GAAGTAGACGGTGAGTGGTTG-3', probe/56-FAM/TTGTAAAGC/Zen/TTCCTCTGGAACTCGCC/3IABkFQ/; CHRFAM7A (chR15:g.30669 904-30670 043) forward 5'-AGTAGCTGGGAC TACAGGTG-3' ${ }^{\prime}$ reverse $5^{\prime}$-ATGTTCCATTTTAGCTATTTTTATGTGTAC-3', probe/56-FAM/CCGGGATGG/Zen/TCTCGATCTCCTGA/3IABkFQ/; duplication region proximal of CHRNA7 triplication (chr15:g.32 024 776-32 024 877) forward $5^{\prime}$-TCTAGTCACTTGGAAACGCC- $3^{\prime}$, reverse $5^{\prime}$-TGCAATTGATA GAGCCACCTG-3', probe/56-FAM/ATGCCCCAG/ZEN/TTGAAATCCACGC/ 3IABkFQ/; and the internal control RPPH1 (chr14:g.20 811 249-20 811332 ), forward 5'-TCTGAATTGGGTTATGAGGTCC-3', reverse GCTTGGAACA GACTCACGG, probe/56-FAM/TCACCTCAG/Zen/CCATTGAACTCACTTCG/ 3IABkFQ/. Each of the reactions contained each primer at $500 \mathrm{nM}, 250 \mathrm{nM}$ probe, 10 ng genomic DNA and $1 \mathrm{X}$ TaqMan Universal PCR Master Mix, no AmpErase UNG (Life Technologies, Carlsbad, CA, USA). The reactions were performed in the StepOnePlus Real Time PCR System (Life Technologies) under the following conditions: AmpliTaq Gold Activation for $10 \mathrm{~min}$ at $95^{\circ} \mathrm{C}$, then 40 cycles of denature $\left(15 \mathrm{sec}, 95^{\circ} \mathrm{C}\right)$ and annealing/extension $(1 \mathrm{~min}$, $60^{\circ} \mathrm{C}$ ). DNA samples III-2, III-3 and III-4 were run in parallel with control genomic DNA for which copy number of all tested regions had previously been demonstrated to be 2 . All reactions were run in quintuplicate.

\section{RESULTS}

CMA performed on DNA obtained from peripheral blood of the index patient (III-2) revealed a triplication at chromosome region $15 q 13.3$, suggested by a $\log$ ratio of $0.7-1.0$. The estimated size of the triplication on the clinical array was a minimum of $0.227 \mathrm{Mb}$, chr15:g.32 218 274-32 445252[3] (hg19) and a maximum of $1.195 \mathrm{Mb}$ with adjacent oligonucleotides showing normal copy number (Figure 2a). The triplication encompasses the entire CHRNA7 gene and the first non-coding exon of a transcript variant of the OTUD7A gene. Interphase FISH analysis performed on cultured lymphocytes of the index patient showed four signals for probe RP11265I17 with three signals clustered together and well separated from the fourth signal, suggesting a triplication rather than a homozygous duplication. FISH analysis on metaphase cells showed no involvement of translocation or insertion of the triplicated region. Subsequent a

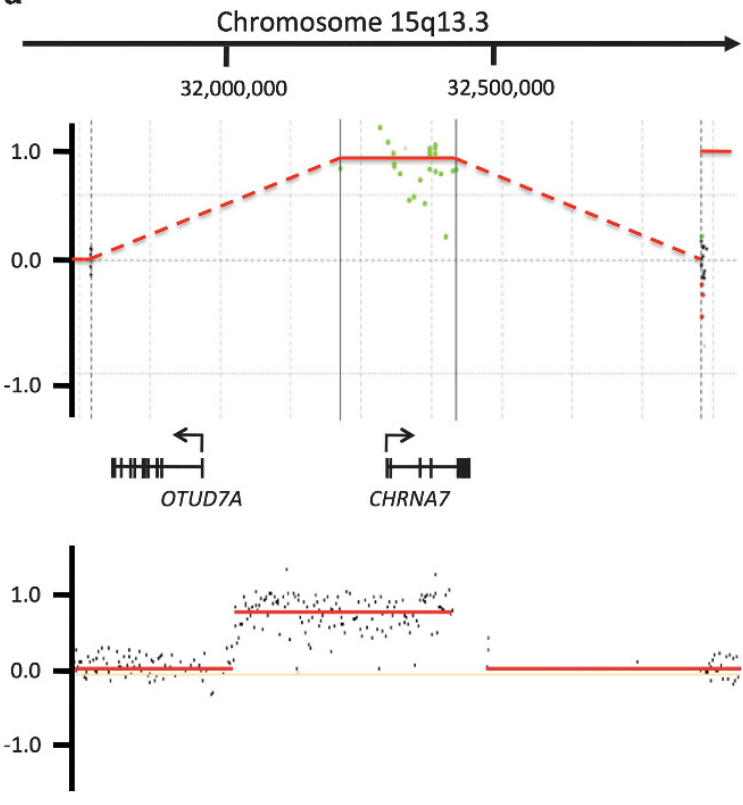

b

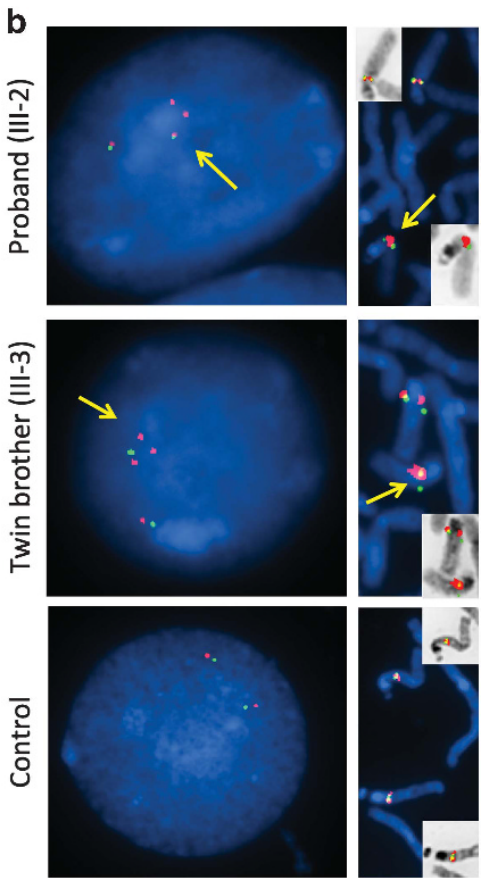

Figure 2 aCGH and FISH analyses. (a) Upper panel: clinical CMA V.8.1 OLIGO (individual III-2), lower panel: NimbleGen $4.2 \mathrm{M}$ array, both showing copy number gain in 15q13.3, encompassing the CHRNA7 gene. Log ratios of 0.7-1.0 suggest triplication of the respective area. (b) FISH analysis, using test probe G248P89177H7 within the first 4 exons of the CHRNA7 gene (red) and control probe G248P85751G8 outside the CHRNA7 gene on 15q13.3 (green). Triplication of 15q13.3 in one copy of chromosome 15, as indicated by yellow arrows, is seen in the proband (III-2, upper panels) and his twin brother (III-3, middle panels), but not in a control individual (lower panels). Left panels: interphase cells, showing three red signals cluster with one green signal, whereas one separate red signal is next to another green signal. Right panels: metaphase cells, showing red signals that are stronger and bigger on one copy of chromosome 15, whereas green signals have comparable size and intensity on both copies of chromosome 15. 
interphase FISH of maternal cells revealed the same CHRNA7 triplication pattern in the proband's mother. Additional investigations revealed two additional triplication carriers in this family: the proband's twin brother (III-3, by chromosome microarray and FISH analysis) and the proband's maternal grandfather (I-1, by FISH analysis). However, CMA and FISH analyses showed that the triplication was not present in the proband's older half-sibling (III-4) who is phenotypically normal. The triplication was not present in the maternal grandmother (I-2), as determined by FISH analysis, either. Additional FISH analyses were performed on both the proband and the twin brother using a fosmid probe G248P89177H7, which is located within a region specific for CHRNA7, and a control probe G248P85751G8 outside of the CHRNA7 gene on 15q13.3 (Figure 2b). The hybridization pattern showed clustering of three CHRNA7 signals and one signal for the control probe in the majority of the interphase cells examined (36/50 interphase cells in subject III-2 and 38/50 interphase cells in subject III-3). Moreover, one chromosome 15 in metaphase cells consistently showed stronger and bigger signals than its homolog. These data are most consistent with the genomic change in this family representing a triplication of CHRNA7 on one copy of chromosome 15 , whereas the other copy of chromosome 15 is normal.

High-resolution oligonucleotide aCGH (4.2 M NimbleGen) confirmed the triplication and enabled further refinement of the genomic coordinates encompassed by the apparent triplication to experimentally allow for better breakpoint mapping (Figure 2a). The proximal breakpoint was amplified using PCR, followed by Sanger sequencing.
It maps to chr15:g.32024752. Using this oligonucleotide array, we were not able to map the precise coordinates of the distal breakpoint, as it falls within the low copy repeats of BP5.

Custom-tiled oligonucleotide aCGH $(4 \times 44 \mathrm{k}$ Agilent $)$ that also covers the LCR region BP4 and BP5 enabled us to map precisely the breakpoint junctions and define the total size of the alteration as $650.4 \mathrm{~kb}$ because it includes part of BP5 $(\sim 233 \mathrm{~kb})$. As a result of the presence of multiple copies of this LCR in the human genome it remains challenging to calculate accurately the copy number gain of that specific region in patients: calculated average $\log _{2}$ ratio of the $233-\mathrm{kb}$ segment is 0.24 consistent with a duplication (considering four copies of BP5 in the reference genome and five copies in subjects with a duplication, expected $\log _{2}$ ratio is 0.32 contrasting with 0.6 , which would be expected if a subject carried six copies). In addition, the array revealed a small duplication preceding the triplication at the proximal breakpoint junction (Figure 3a), which we were able to further characterize by long-range PCR using primers designed at both copy number transition sites (Figure 3b). That DUP-TRP breakpoint junction unveiled the presence of an inversion, which indicated that the triplicated segment is in an inverted orientation with respect to the duplication. Therefore, the rearrangement present in the patients described herein appears to be best classified as a DUP-TRP/INV-DUP type, ${ }^{12}$ although we were not able to sequence the distal breakpoint junction to define whether an inverted repeat had mediated that rearrangement or not.

Real-time qPCR was performed in order to distinguish copy number of CHRNA7 and CHRFAM7A, the latter of which is a

a

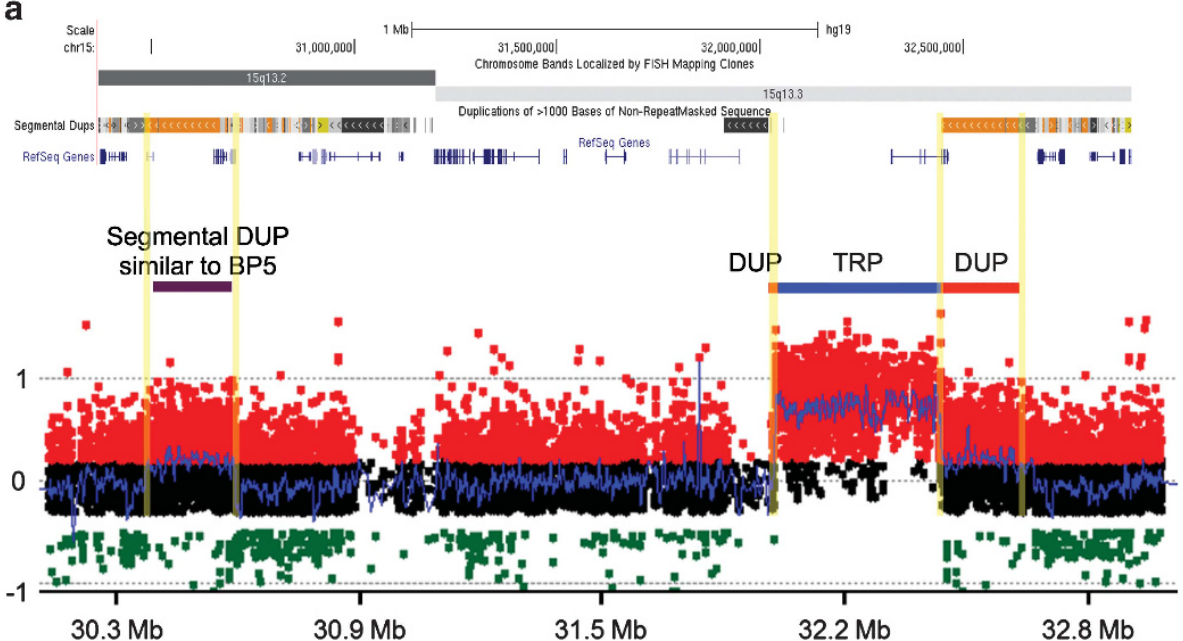

b DUP-TRP/INV junction

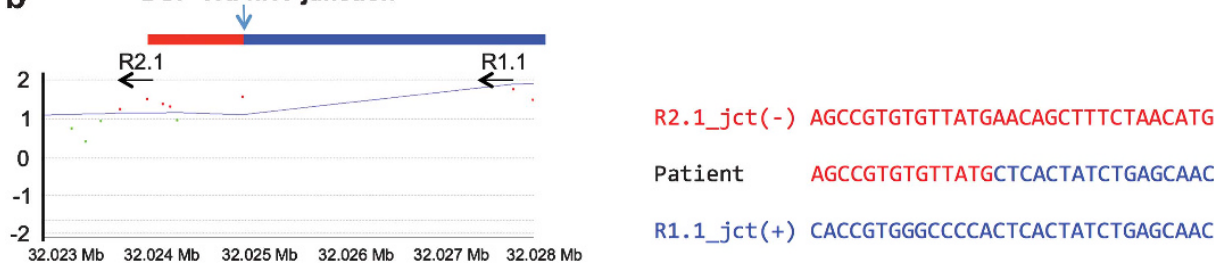

Figure 3 Agilent high-resolution tiling-path aCGH. (a) CNV results for individual III-2, shown in the context of the genomic human reference (hg19). LCRs present within the region color coded according to nucleotide similarity as presented in UCSC genome browser (orange >99\%, light to dark grey 90-98\% similarity). The triplicated segment is represented by a blue rectangle; duplicated segment is represented by red rectangles. Note that BP4 seems duplicated likely due to the high similarity between probes that span BP4 and BP5 (purple rectangle). (b) Close view of aCGH proximal DUP-TRP breakpoint showing location of primers used to amplify and sequence the junction (sequence is shown on the aCGH view to the right). Of note, primers R2.1 and R1.1 have the same orientation in the reference genome but produce a patient-specific PCR product that reveals that the triplication is inverted in orientation regarding the small duplication. Sequencing data is color-coded to show transition from the duplicated to the triplicated segment. 
human-specific fusion gene at 15q13.2 sharing exons 5-10 with CHRNA7. The analysis was carried out using genomic DNA of individuals III-2, III-3, III-4 and a male control known to have two copies of each CHRFAM7A and CHRNA7. Data as shown in Figure 4 suggest that both the proband and his twin brother have four copies of CHRNA7 and one copy of CHRFAM7A. In addition, qPCR confirmed the presence of the small duplication proximal to the CHRNA7 triplication in both twins. The proband's older half-sibling has normal copy number of CHRNA7, as had been suggested by CMA and FISH.

\section{DISCUSSION}

This is the first case report of CHRNA7 triplication, with four individuals in three generations being affected. Although deletions of CHRNA7 cause ID and neuropsychiatric phenotypes with relatively high penetrance, ${ }^{3,4,6}$ the pathogenicity of CHRNA7 duplications remains unclear, given its high prevalence in the general population with multiple individuals that are clinically unaffected. ${ }^{7}$ Therefore, it has been uncertain whether CHRNA7 is a dosage-sensitive gene with penetrant clinical phenotypes for both deletions and duplications.

In this report, the family members harboring the triplication are affected with cognitive abilities in the borderline intellectual functioning range, and various degrees of neuropsychiatric pathology. Both the proband and his twin brother are affected with PDD-NOS, dysthymia, and anxiety disorder. Their mother has been diagnosed with bipolar disorder, anxiety disorder, attention deficit disorder, learning disability, and seizures since her twenties. Although information about the maternal grandfather is limited, he was reported as affected with alcoholism, drug addiction, and displays abusive behaviors to others, including his family - neurobehavioral traits observed and delineated in association with the initial deletion described at this locus. ${ }^{1}$ The variability of expressivity seen in the family members with CHRNA7 triplication is similar to what has been reported in individuals with CHRNA7 duplication and many other CNVs associated with neuropsychiatric phenotypes. ${ }^{13}$ On the other hand, at least in this family, and to the point studied, there is a

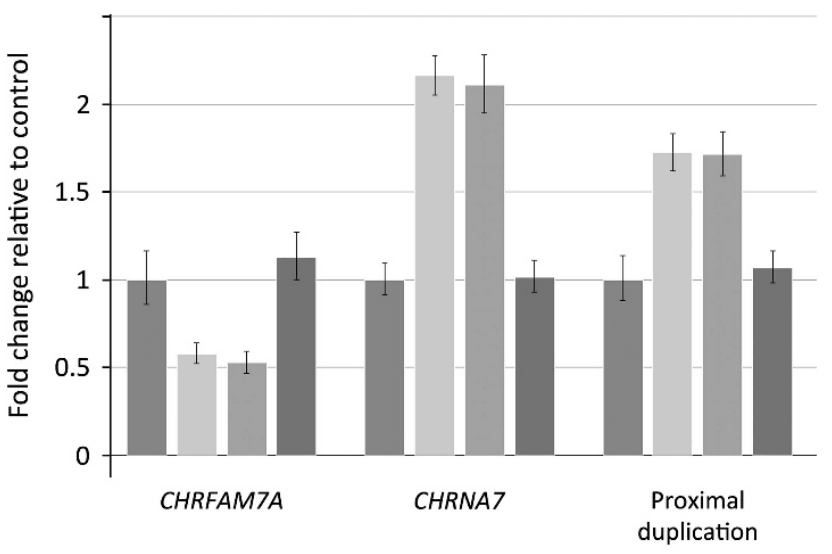

control

III-2 III-3

III-4

Figure 4 Real-time quantitative PCR of genomic DNA. Three primer and probe sets were used, unique to CHRNA7, CHRFAM7A, and the small duplication proximal to CHRNA7, respectively. Data were normalized to an internal control, RPPH1 on chromosome 14 , and are shown relative to a control individual known to carry two copies of each CHRNA7 and CHRFAM7A. Reactions were run in quintuplicate. Error bars indicate SD. complete penetrance of behavioral phenotypes for the CHRNA7 triplication. Whether this holds true for CHRNA7 triplications in general remains to be seen and awaits the identification of additional cases and families. Nevertheless, precedence exist for penetrance of neuropsychiatric traits being related to further in increments of gene dosage; triplications at the STS locus appear to be more penetrant than duplications for a DD/ID-associated phenotype. ${ }^{14}$

Triplications are very rare chromosomal rearrangements. ${ }^{15}$ In general, the resulting clinical phenotype is consistent with, but more severe than, the duplication of the same locus. ${ }^{12,14,16-18}$ Triplications of genomic loci associated with neurocognitive and neuropsychiatric abnormalities have been described, among others, at $22 \mathrm{q} 11.2,{ }^{18} 7 \mathrm{p} 11.2,{ }^{19} \mathrm{Xq} 28$ encompassing the MECP2 gene, ${ }^{20}$ and 7 q11.23 at the Williams syndrome critical region. ${ }^{17}$ Triplications can, in general, be classified as types I and II, as well as being recurrent or non-recurrent, which relates to the molecular mechanism underlying their formation. ${ }^{21}$ Type I may result from a two-step NAHR involving flanking paralogous LCRs such as those producing triplications of the STS region on $\mathrm{Xp} 22.31,{ }^{14}$ whereas the type II triplication product displays an unusual DUP-TRP/INV-DUP pattern of rearrangement it has been reported in $20 \%$ of the MECP2 CNV gains at Xq28, and also observed at the PLP1 locus at Xq22. ${ }^{12,22}$ Inverted repeats in the human genome appear to be a susceptibility architecture for type II triplication. ${ }^{23}$ Remarkably, LCRs BP4 and BP5, which mediate most of the rearrangements involving CHRNA7, are constituted by a complex set of large duplicated segments with $>99 \%$ identity, lying in an inverted orientation relative to each other (Figure 3a). ${ }^{24}$ Polymorphic inversions mediated by BP4 and BP5 are frequently found in individuals from various populations ${ }^{24}$ and have been hypothesized to predispose to microdeletions and microduplications of the region by NAHR. In fact, at least six classes of microduplications, varying in size from $350 \mathrm{~kb}$ to $1.6 \mathrm{Mb}$, were shown to arise involving pre-existing heterogeneous inverted BP4 and BP5 chromosomes. ${ }^{7}$ Here we describe a non-recurrent triplication of $650.4 \mathrm{~kb}$, for which the breakpoint junctions do not involve LCRs (Figure 3b), implicating an alternate underlying mechanism of formation. Its molecular characterization enabled us to experimentally classify it as type II or DUP-TRP/INV-DUP, likely generated by a replication-based mechanism. ${ }^{25}$

The nervous system is exquisitely sensitive to the dosage of many proteins. Interestingly, there exist multiple examples for which both the deletion and duplication cause overlapping, if not similar, clinical phenotypes, a classic example is the myelinopathy phenotypes associated with CMT1A duplication and HNPP deletion. ${ }^{26}$ It has been speculated that the similar cognitive and social behavior phenotypes caused by both loss and gain of function mutations is the manifestation of a failure in neuronal homeostatic response because of downstream effect of various molecular changes. ${ }^{27}$

However, there have also been many examples of 'mirror traits' associated with gain versus loss at a locus. Perhaps the best classified is microcephaly versus macrocephaly at the 16 p11.2 locus, associated with loss versus gain, respectively, and at the 1q21.1 locus, associated with gain versus loss, respectively. ${ }^{28}$ Remarkably, such observations have been used to support a prominent theory in the social anthropology literature by $\mathrm{Crespi}^{29}$ proposing that autism and schizophrenia represent opposing extreme phenotypes of social behavior.

This case report of the CHRNA7 triplication family supports the idea of dosage sensitivity for CHRNA7 encoding the alpha7 nicotinic acetylcholine receptor, which forms homopentameric ligand-gated cation channels, highly expressed at the synapse and elsewhere in the 
brain. ${ }^{30}$ Although overexpression of other nicotinic acetylcholine receptor subunits has been shown to modify and cause abnormal behaviors, ${ }^{31}$ this has not conclusively been proven for CHRNA7. Given the frequency of CHRNA7 duplications, even a modest penetrance of $5-10 \%$ would entail significant pathogenic potential in the population overall. Studies investigating this, as well as potential genetic and environmental modifiers, are even more important, given that the alpha7 nicotinic receptor represents an excellent target for therapeutic intervention. ${ }^{32}$ The study of large cohorts of individuals for sequence variants, mutations and CNVs of CHRNA7 gene are warranted to be able to elucidate a more accurate role in health and disease.

\section{URLs}

Leiden Open Variation Database (LOVD), http://www.lovd.nl/; CHRNA7-specific variations listed in LOVD, http://databases. lovd.nl/shared/genes/CHRNA7.

\section{CONFLICT OF INTEREST}

Drs Carvalho, Lupski, Stankiewicz, Cheung, Bi and Schaaf are faculty members of the Department of Molecular and Human Genetics at Baylor College of Medicine, which derives revenue from the chromosomal microarray analysis offered in the Medical Genetics Laboratory.

\section{ACKNOWLEDGEMENTS}

We are indebted to the family who participated in this study. Dr Schaaf's work is generously supported by the Joan and Stanford Alexander Family. Dr Schaaf is also a recipient of a Doris Duke Clinical Scientist Development Award. NIH grant HD037283 supported Dr Ge's work in the laboratory of Dr Arthur Beaudet. In addition, this work was supported in part by US National Institute of Neurological Disorders and Stroke grant NS058529 to JRL.

1 Ben-Shachar S, Lanpher B, German JR et al: Microdeletion 15q13.3: a locus with incomplete penetrance for autism, mental retardation, and psychiatric disorders. J Med Genet 2009; 46: 382-388.

2 van Bon BW, Mefford HC, Menten B et al: Further delineation of the $15 q 13$ microdeletion and duplication syndromes: a clinical spectrum varying from nonpathogenic to a severe outcome. J Med Genet 2009; 46: 511-523.

3 Shinawi M, Schaaf CP, Bhatt SS et al: A small recurrent deletion within $15 q 13.3$ is associated with a range of neurodevelopmental phenotypes. Nat Genet 2009; 41 1269-1271.

4 Cooper GM, Coe BP, Girirajan S et al: A copy number variation morbidity map of developmental delay. Nat Genet 2011; 43: 838-846.

5 Sahoo T, Theisen A, Rosenfeld JA et al: Copy number variants of schizophrenia susceptibility loci are associated with a spectrum of speech and developmental delays and behavior problems. Genet Med 2011; 13: 868-880.

6 Helbig I, Mefford HC, Sharp AJ et al: 15q13.3 microdeletions increase risk of idiopathic generalized epilepsy. Nat Genet 2009; 41: 160-162.

7 Szafranski P, Schaaf CP, Person RE et al: Structures and molecular mechanisms for common 15q13.3 microduplications involving CHRNA7: benign or pathological? Hum Mutat 2010; 31: 840-850.
8 Williams NM, Franke B, Mick E et al: Genome-wide analysis of copy number variants in attention deficit hyperactivity disorder: the role of rare variants and duplications at 15q13.3. Am J Psychiatry 2012; 169: 195-204.

9 Boone PM, Bacino CA, Shaw CA et al: Detection of clinically relevant exonic copy-number changes by array CGH. Hum Mutat 2010; 31: 1326-1342.

10 Carvalho $\mathrm{CM}$, Zhang $\mathrm{F}$, Liu $\mathrm{P}$ et al: Complex rearrangements in patients with duplications of MECP2 can occur by fork stalling and template switching. Hum $\mathrm{Mo}$ Genet 2009; 18: 2188-2203

11 Shinawi M, Liu P, Kang SH et al: Recurrent reciprocal 16p11.2 rearrangements associated with global developmental delay, behavioural problems, dysmorphism, epilepsy, and abnormal head size. J Med Genet 2010; 47: 332-341.

12 Carvalho CM, Ramocki MB, Pehlivan D et al: Inverted genomic segments and complex triplication rearrangements are mediated by inverted repeats in the human genome. Nat Genet 2011; 43: 1074-1081.

13 Sebat J, Levy DL, McCarthy SE: Rare structural variants in schizophrenia: one disorder multiple mutations; one mutation, multiple disorders. Trends Genet 2009; 25 528-535.

14 Liu P, Erez A, Nagamani SC et al: Copy number gain at Xp22.31 includes complex duplication rearrangements and recurrent triplications. Hum Mol Genet 2011; 20 1975-1988.

15 Reddy KS, Logan JJ: Intrachromosomal triplications: molecular cytogenetic and clinical studies. Clin Genet 2000; 58: 134-141.

16 Bi W, Sapir T, Shchelochkov OA et al: Increased LIS1 expression affects human and mouse brain development. Nat Genet 2009; 41: 168-177.

17 Beunders G, van de Kamp JM, Veenhoven RH, van Hagen JM, Nieuwint AW, Sistermans EA: A triplication of the Williams-Beuren syndrome region in a patient with mental retardation, a severe expressive language delay, behavioural problems and dysmorphisms. J Med Genet 2010; 47: 271-275.

18 Yobb TM, Somerville MJ, Willatt L et al: Microduplication and triplication of 22q11.2 a highly variable syndrome. Am J Hum Genet 2005; 76: 865-876.

19 Girirajan S, Williams SR, Garbern JY, Nowak N, Hatchwell E, Elsea SH: 17p11.2p12 triplication and del(17)q11.2q12 in a severely affected child with dup(17)p11.2p12 syndrome. Clin Genet 2007; 72: 47-58.

20 del Gaudio D, Fang P, Scaglia F et al: Increased MECP2 gene copy number as the result of genomic duplication in neurodevelopmentally delayed males. Genet Med 2006; 8: 784-792.

21 Liu P, Carvalho CM, Hastings PJ, Lupski JR: Mechanisms for recurrent and complex human genomic rearrangements. Curr Opin Genet Dev 2012; 22 211-220.

22 Shimojima K, Mano T, Kashiwagi M et al: Pelizaeus-Merzbacher disease caused by a duplication-inverted triplication-duplication in chromosomal segments including the PLP1 region. Eur J Med Genet 2012; 55: 400-403.

23 Dittwald P, Gambin T, Gonzaga-Jauregui C et al: Inverted low-copy repeats and genome instability-a genome-wide analysis. Hum Mutat 2013; 34: 210-220.

24 Sharp AJ, Mefford HC, Li K et al: A recurrent 15q13.3 microdeletion syndrome associated with mental retardation and seizures. Nat Genet 2008; 40 : 322-328.

25 Hastings PJ, Lupski JR, Rosenberg SM, Ira G: Mechanisms of change in gene copy number. Nat Rev Genet 2009; 10: 551-564.

26 Schaaf CP, Wiszniewska J, Beaudet AL: Copy number and SNP arrays in clinica diagnostics. Annu Rev Genomics Hum Genet 2011; 12: 25-51.

27 Zoghbi HY, Warren ST: Neurogenetics: advancing the "next-generation" of brain research. Neuron 2010; 68: 165-173.

28 Lupski JR: Brain copy number variants and neuropsychiatric traits. Biol Psychiatry 2012; 72: 617-619.

29 Crespi BJ: Revisiting Bleuler: relationship between autism and schizophrenia. $\mathrm{Br}$ Psychiatry 2010; 196: 495; author reply 495-496.

30 Lin H, Vicini S, Hsu FC et al: Axonal alpha7 nicotinic ACh receptors modulate presynaptic NMDA receptor expression and structural plasticity of glutamatergic presynaptic boutons. Proc Natl Acad Sci USA 2010; 107 $16661-16666$

31 Vinals X, Molas S, Gallego X et al: Overexpression of alpha3/alpha5/beta4 nicotinic receptor subunits modifies impulsive-like behavior. Drug Alcohol Depend 2012; 122 247-252.

32 Kem WR: The brain alpha7 nicotinic receptor may be an important therapeutic target for the treatment of Alzheimer's disease: studies with DMXBA (GTS-21). Behav Brain Res 2000; 113: 169-181. 